\title{
Gestión de la tecnología: estructura intelectual de las investigaciones de la última década
}

\section{Technology management: intellectual structure of research in the last decade}

\author{
Hugo Martínez \\ Ingeniero Electrónico, estudiante de Doctorado en Ingeniería. Investigador de la \\ Universidad Industrial de Santander. Bucaramanga, Colombia. \\ Contacto: hugo.martinez@correo.uis.edu.co
}

Edna R. Bravo

Ingeniera Industrial, doctora en Administración de Empresas. Docente de la Universidad Industrial de Santander. Bucaramanga, Colombia.

Contacto:erbravoi@uis.edu.co

\section{Luis Eduardo Becerra Ardila}

Ingeniero Industrial, magister en Administración. Docente de la Universidad Industrial de Santander. Bucaramanga, Colombia. Contacto: lbecerra@uis.edu.co

Fecha de recepción: 30 de noviembre de 2011

Clasificación del artículo: Revisión

Fecha de aceptación: 16 de octubre de 2012

Financiamiento: Universidad Industrial de Santander

Palabras clave: bibliometría, gestión de la tecnología, investigación.

Key words: bibliometric, technology management, research.

\section{RESUMEN}

El propósito de este artículo es caracterizar mediante herramientas bibliometrícas el desarrollo intelectual de la disciplina Gestión de la Tecnología, identificado los patrones de productividad científica y los temas de investigación clave durante la última década. La metodología utilizada consistió en tres fases: la recolección de información relevante del Sciences Citation Index (SCI) del Institute for Scientific Information (ISI), el análisis de la información por medio del análisis estructurado de palabras clave y la generación de resultados. Los principales resultados muestran que existen temas de investigación emergentes que mejoran y operacionalizan las teorías sobre Gestión de la Tecnología. Entre estos temas se encuentran: e-learning, librerías digitales, cambio climático, intercambio electrónico de datos, prácticas organizativas y orientación al cliente. 


\section{ABSTRACT}

The purpose of this paper is to characterize the intellectual development of a topic called Technology management by using bibliometric tools. The idea is to identify patterns and key research topics during the last decade. The methodology used consists of three major phases, namely the collection of relevant information from the Science Citation Index (SCI) at the Institute for
Scientific Information (ISI), the transformation of information by descriptive and co-occurrence analysis, and the gathering of results. The main results show emerging research topics such as elearning, digital libraries, climate change, electronic data exchange, organizational practices, and customer orientation; which we believe should improve the application of Technology management theories.

\section{INTRODUCCIÓN}

La gestión de la tecnología se ha consolidado en las últimas décadas como una disciplina de gran interés para la comunidad científica [1]. Este interés puede ser explicado por la explosión tecnológica del último siglo [2]; además de la modernización y la rápida difusión de las investigaciones científicas. La literatura en esta disciplina ha mostrado un acelerado crecimiento durante la última década. Lo anterior, ha impulsado la creación de revistas científicas que sirven de herramientas para la difusión de investigaciones asociadas a la disciplina. En estudios previos como el de Linton [3], se identificaron las principales revistas científicas como: IEEE Transactions on Engineering Management, Technology Forecasting and Social Change, $R \& D$ Management, Research Policy, Technovation y Journal of Engineering and Technology Management. Las investigaciones en gestión de la tecnología son de carácter interdisciplinar, por la combinación de tópicos como las ciencias, la ingeniería, la gestión y los negocios.

Por otra parte, en las ciencias de la información y librería (The library and information science -LIS) existen técnicas y métodos como la bibliometría, que permiten el análisis de aspectos cuantitativos de la información con el objetivo de mejorar el entendimiento de los procesos de comunicación y el enfoque evolutivo de una disciplina [4]. Estas técnicas bibliométricas son aplicadas generalmente en: la identificación de tendencias, descripciones de la productividad científica, crecimiento de las publicaciones e identificación de revistas científicas clave [5]. Por medio de estas técnicas, los patrones de las publicaciones científicas son analizados generalmente como datos integrados. Incluida en estas técnicas, el análisis de co-ocurrencia de palabras clave tiene como objetivo la evaluación de la fortaleza de los enlaces entre documentos, utilizando como indicador las palabras clave propuestos por los autores de las investigaciones [6].

Previamente, en la disciplina de gestión de la tecnología, se han realizado estudios bibliométricos como: análisis de tecnologías emergentes [7], innovación de productos [8], gestión de programas [9] y tendencias futuras de investigación [10]. Otros estudios bibliométricos se han orientado hacia tecnologías específicas como: energía de hidrogeno [11], súper condensadores [12], biomedicina [13] e inteligencia computacional [14].

Sin embargo, no existe un análisis bibliométrico de la disciplina de gestión de la tecnología que 
proporcione una visión holística de la estructura intelectual de las investigaciones de los últimos años. Este estudio, basándose en trabajos como el de Pilkington [1], intenta mejorar y actualizar los resultados de las investigaciones. Por tanto, surge la oportunidad de realizar un estudio bibliométrico de la disciplina Gestión de la Tecnología, que permita describir el estado de arte actual y la estructura intelectual resultante de las investigaciones desarrolladas por la comunidad académica durante la última década. Por ello, el propósito de este estudio es caracterizar mediante herramientas bibliométricas el desarrollo intelectual de la disciplina Gestión de la Tecnología, identificado los patrones de productividad científica y los tópicos de investigación clave y emergente durante los años 2001-2010.

\section{APLICACIÓN DE TÉCNICAS DE BIBLIOMETRÍA: DESCRIPCIÓN Y ANÁLISIS DE DOMINIOS DE CONOCIMIENTO}

La bibliometría es un campo de investigación que emergió con importantes investigadores como Garfield [15] y De Solla [16]. Los análisis bibliométricos se fundamentan en la información incluida en los campos bibliográficos de los artículos científicos [17], [18]. La bibliometría puede ser entendida como el análisis cuantitativo con el uso de métodos matemáticos (especialmente estadísticos) de las características bibliográficas de un conjunto de conocimientos representado en libros y otros medios escritos [19], [20]. El objetivo general de las técnicas bibliométricas es la construcción de estructuras cognitivas de un campo, utilizando los términos de autores, revistas científicas, países, temas clave y colaboración entre autores.

Los análisis cuantitativos, aplicando técnicas bibliométricas, han permitido sobrepasar distorsiones derivadas de la subjetividad en contextos de valoración [21]. También han descrito propie- dades internas y externas en campos o dominios científicos [22]. La bibliometría se basa en la premisa que los investigadores construyen sobre el trabajo de otros investigadores, asumiendo que los artículos son reflexiones del conocimiento producido por la investigación científica [14]. Los métodos, principios y técnicas relacionados a la bibliometría han sido aplicados en diferentes disciplinas de ciencia e ingeniería [23], [24]. Incluso han sido aplicados en industrias específicas como biotecnología y nanotecnología [25].

Una técnica bibliométrica muy utilizada es el análisis de co-ocurrencias de palabras clave. Estos análisis de co-ocurrencia de palabras clave se utilizan generalmente en la representación gráfica de diferentes disciplinas y campos de investigación [26] - [31]. Sin embargo, el análisis de copalabras también ha sido utilizado para investigar otros fenómenos como: generación de hipótesis [32], descubrimiento de conocimiento [33], clasificación de artículos [34], dinámica de investigación científica [35], relaciones inter organizativas [36] y en el análisis de patentes [37]. La técnica bibliométrica de copalabras es apropiada para la representación del mapa de la estructura de conocimiento del área Gestión de la Tecnología, con el fin de obtener los temas clave, sus relaciones e importancia encontrados en la literatura en la última década (2001-2010).

Una Técnica para la elaboración del mapeo de los datos resultantes del análisis de co-ocurrencia de palabras clave es la Visualización de Similaridades-VOS [38]. Esta técnica permite visualizar similitudes entre objetos en un espacio bidimensional, convirtiéndose en una alternativa a la conocida técnica Escalamiento Multidimensional-MDS [39]. En este estudio, la técnica VOS utiliza las palabras clave incluidas en cada uno de los registros como objetos de análisis o interés. Por tanto, la co-ocurrencia de palabras clave se aplica para formar una gráfica bidimensional de las proximidades entre palabras. Debido a que las 
palabras son la base de conceptos científicos, su separación espacial permite visualizar la distancia conceptual y la investigación conjunta entre palabras en un dominio determinado.

\section{METODOLOGÍA}

El diseño metodológico utilizado está compuesto por tres grandes fases con el fin de alcanzar los objetivos de esta investigación: 1) la recolección de información relevante de las bases de datos del Instituto de Información Científica - ISI, 2) la transformación y análisis de información aplicando técnicas bibliométricas y, finalmente, 3) la generación de resultados.

En la primera fase se utilizó la base de datos del Índice de Citación de Ciencias Sociales (SSCI) del Instituto de Información Científica (ISI) para extraer la información referente a los registros científicos durante los años 2001-2010. La búsqueda se realizó utilizando la ecuación "technolog* AND management" en el campo 'tópico' de la ISI Web of Science, obteniendo un total de 10678 registros. Estos registros incluían artículos, revisiones, memorias de congresos, material editorial, revisiones de libros, entre otros. Del total de registros se seleccionaron 9570 artículos y 775 revisiones. Este filtro se aplicó con el objetivo de centrar la investigación en revisiones y estudios de alta calidad que han sido revisados por pares. Al final, la base de datos se redujo a 10345 documentos entre artículos y revisiones, tomados como unidad de estudio para la fase de análisis y transformación de datos. Cada uno de los artículos encontrados fue almacenado con los diferentes campos a analizar: nombres de los autores, afiliación, país de origen, revistas en las que se publica, año de publicación, referencias y palabras clave. Estos campos bibliográficos son el insumo para la segunda fase de trasformación y análisis de la información aplicando técnicas bibliométricas.
La fase de transformación y análisis de datos se dividió en dos partes: la primera, relacionada a la estadística descriptiva basada en la frecuencia del conteo de cada uno de los campos bibliográficos de los artículos analizados. Esta parte descriptiva muestra tendencias e indicadores generales que han sido utilizados en otros estudios de: campos bibliográficos de autores [40], países [41], revistas científicas [42], instituciones [43], áreas de investigación [44]; y crecimiento productivo general del campo.

Además del análisis estadístico descriptivo de la disciplina de Gestión de la Tecnología; también se hizo un análisis bibliométrico con la técnica de análisis de co-ocurrencia de palabras clave. Este tipo de análisis realiza el conteo y estudio del número de veces que aparecen los pares de palabras clave en un mismo artículo, generando como resultado un cuadro con los tópicos investigados en la literatura científica seleccionada [28]. El análisis de co-ocurrencia, considera que las palabras clave constituyen una descripción adecuada del contenido o enlaces entre los problemas planteados por los artículos [27]; por tanto, pueden ser utilizadas como indicadores de los términos clave en un dominio de conocimiento específico [45].

La organización de la información de palabras clave se realizó mediante la utilización del aplicativo SITKIS [46]. Esto implica, la selección de las 100 palabras clave más utilizadas en el área (es decir aquellas con mayor frecuencia de aparición en los artículos). En este proceso se empleó una rutina específica de SITKIS para hacer un análisis de relaciones entre las diferentes palabras clave almacenadas en la base de datos de información bibliográfica. Posteriormente, se generó un archivo con estas relaciones, almacenándolas en una base de datos ACCES de Microsoft. Finalmente, se obtuvo la matriz de co-ocurrencia de palabras clave de esta base de datos. Esta matriz describe el número de veces que pares de palabras clave aparecen en los artículos analizados y sirve como 
insumo para realizar un mapeo del espacio de conocimiento del área Gestión de la Tecnología.

El método de mapeo de datos fue realizado utilizando la técnica de visualización de Similaridades-VOS [41]. Esta técnica es una forma alternativa a la técnica de escalamiento multidimensional-MDS [39]; para visualizar la similaridad entre objetos. En este caso, las palabras clave de autores, son utilizadas como conceptos científicos para visualizar la distancia conceptual de la investigación en el domino de Gestión de la Tecnología. El método VOS fue aplicado porque los mapas construidos con esta técnica tienen una mejor representación del conjunto de datos que los resultantes de la aplicación del MDS [47]. Además, este método ha sido utilizado anteriormente por otros estudios que han obtenido resultados óptimos [48].

La tercera fase proporciona una descripción general de los factores clave de la investigación, los temas emergentes, futuras oportunidades y relaciones entre temas de investigación, como se analizará en la próxima sección de resultados.

\section{RESULTADOS}

El propósito de este estudio fue analizar las publicaciones realizadas en revistas científicas incluidas en las bases de datos del Instituto de Información Científica -ISI Web of Science- durante los años 2001-2010 sobre el tópico "Gestión de la Tecnología". Los análisis incluyen una descripción general de la productividad científica de esta disciplina en el ámbito global. Además de una descripción detallada de la técnica de co-ocurrencia de palabras clave, con el fin de entender la estructura de conocimiento de las investigaciones en Gestión de la Tecnología, sus patrones y tendencias asociadas.

\subsection{Descripción general de la productividad científica}

Como es mostrado en la figura 1, la disciplina Gestión de la Tecnología ha tenido un incremento en los artículos publicados de más del $300 \%$. Este dato es obtenido de la comparación del número de artículos del año 2010 (1849 artículos) y el año

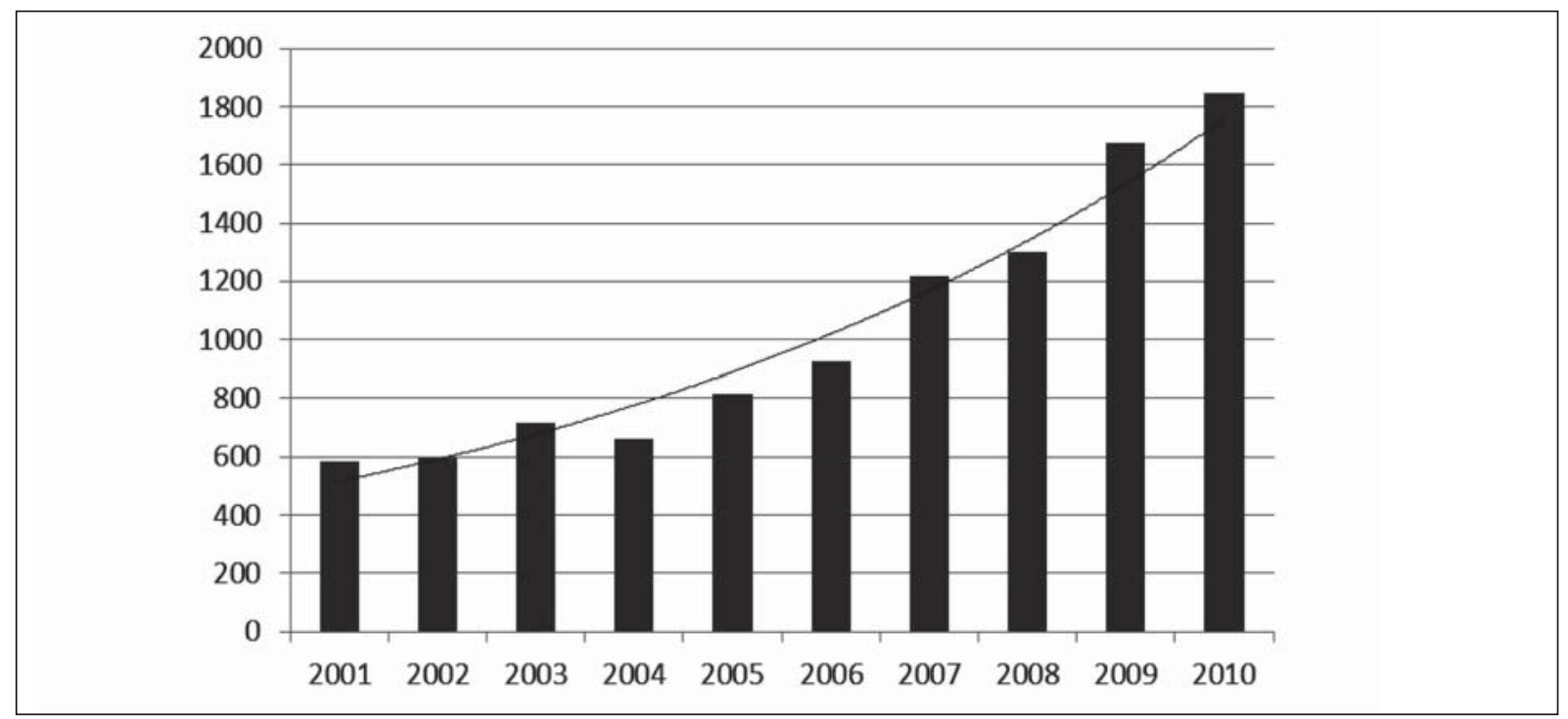

Figura 1. Distribución y tendencia de la productividad científica en el área de Gestión de la Tecnología Fuente: elaboración propia. 
2001 (586 artículos). La tasa de productividad ha tenido un crecimiento promedio del $14 \%$ entre años contiguos. Sin embargo, en el año 2004 se presentó una reducción del 7,5\% en el número de artículos publicados. Los años con mayor tasa de productividad han sido 2008 y 2006, con $29 \%$ y $31 \%$ respectivamente.

En lo referente a las subáreas más estudiadas de la disciplina Gestión de la Tecnología durante la última década se destacan: economía y negocios (4720 artículos), ciencias de la computación (1780 artículos), ingeniería (1715 artículos), ciencias de la información y librería (1554 artículos) e investigación de operaciones y ciencias de la gestión (1195 artículos). En general, basados en los datos de los últimos años, se espera que la tendencia de la productividad científica sobre el tópico "gestión de la tecnología" sea creciente en los próximos años, debido al interés que éste ha despertado en la comunidad científica. Lo anterior, puede motivar a los jóvenes investigadores al desarrollo de nuevos estudios en el área. Los tres principales idiomas en que han sido escritas las publicaciones sobre Gestión de la Tecnología son: inglés $(96 \%)$, español $(1,2 \%)$ y portugués $(0,7 \%)$.

Los resultados del estudio bibliométrico muestran un total de 119 países con al menos una publicación sobre gestión de la tecnología. En la tabla 1, se muestran los 20 países con mayor productividad en el área. La contribución de cada país fue estimada por el lugar de afiliación de los diferentes autores incluidos en cada publicación. La mayoría de países mostrados en la tabla 1 , son de origen europeo, asiático y norte americano. También, se pudo comprobar que el único país Latinoamericano con un número superior a 100 publicaciones, en el tópico de Gestión de la Tecnología, es Brasil; además, se observa claramente la ausencia de países Africanos. Países pertenecientes al G8 como: Estados Unidos, Canadá, Francia, Alemania, Italia, Inglaterra y Japón tienen una participación notable en la lista de los países productivos debido a su elevado número de registros. USA, Inglaterra y Canadá producen el $50 \%$ de los artículos sobre gestión de la tecnológica. En Latinoamérica los países más productivos son, en orden descendente: Brasil (127 registros), Méxi-

Tabla 1. Lista de países más productivos

\begin{tabular}{cc|cc}
\hline Número de Registros & Países & $\begin{array}{c}\text { Número de } \\
\text { Registros }\end{array}$ & Países \\
\hline 4311 & Estados & $\mathbf{2 1 0}$ & Italia \\
$\mathbf{1 2 4 4}$ & Unidos & $\mathbf{2 0 9}$ & Francia \\
$\mathbf{5 8 0}$ & Canglaterra & $\mathbf{1 8 3}$ & Suecia \\
$\mathbf{5 1 5}$ & Taiwán & $\mathbf{1 7 1}$ & Escocia \\
$\mathbf{5 1 1}$ & Australia & $\mathbf{1 5 0}$ & Finlandia \\
$\mathbf{4 4 0}$ & Holanda & $\mathbf{1 5 0}$ & Singapur \\
$\mathbf{4 2 8}$ & China & $\mathbf{1 4 2}$ & Suiza \\
$\mathbf{3 9 4}$ & España & $\mathbf{1 3 5}$ & Noruega \\
$\mathbf{3 5 9}$ & Alemania & $\mathbf{1 3 2}$ & Japón \\
$\mathbf{2 2 3}$ & Corea del Sur & $\mathbf{1 2 7}$ & Brasil \\
\hline
\end{tabular}




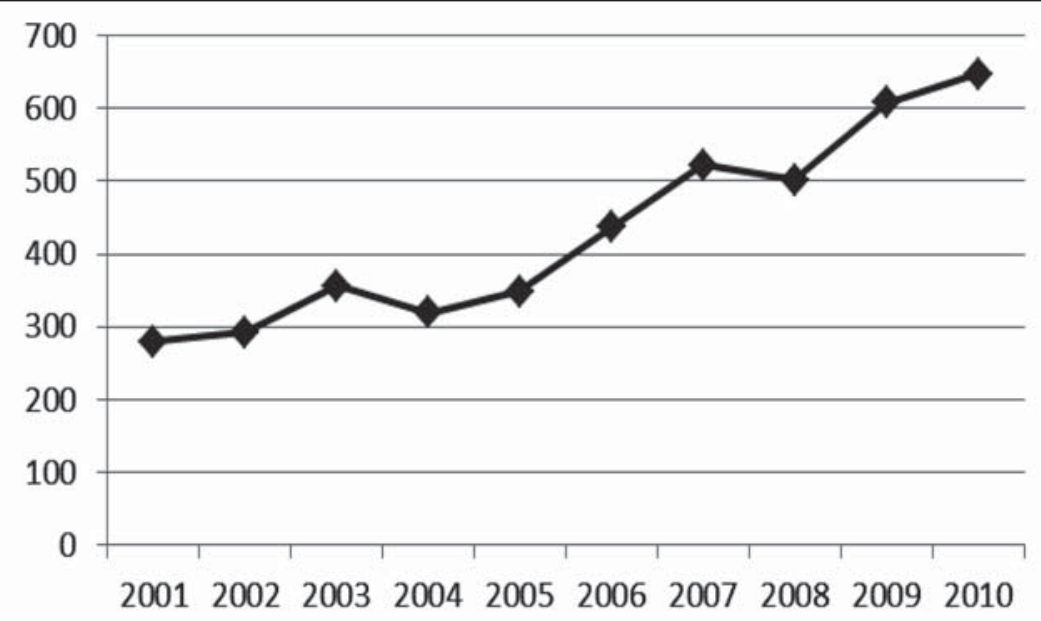

Figura 2. Evolución de la productividad científica de Estados Unidos, 2001-2010

Fuente: elaboración propia.

co (27 registros), Chile (20 registros), Argentina (16 registros), Venezuela (15 registros) y Colombia (11 registros). El número de publicaciones derivadas de las investigaciones se consideran como un indicador fiable que refleja la actividad y nivel académico de los actores en determinado tópico de interés [49].
La figura 2 muestra la productividad científica de Estados Unidos. La figura 3 muestra la productividad científica de los siguientes cuatro países más productivos, es decir: Inglaterra, Canadá, Taiwán y Australia. En un principio, se había planificado representar los cinco países más productivos en el área en una misma figura, sin embargo,

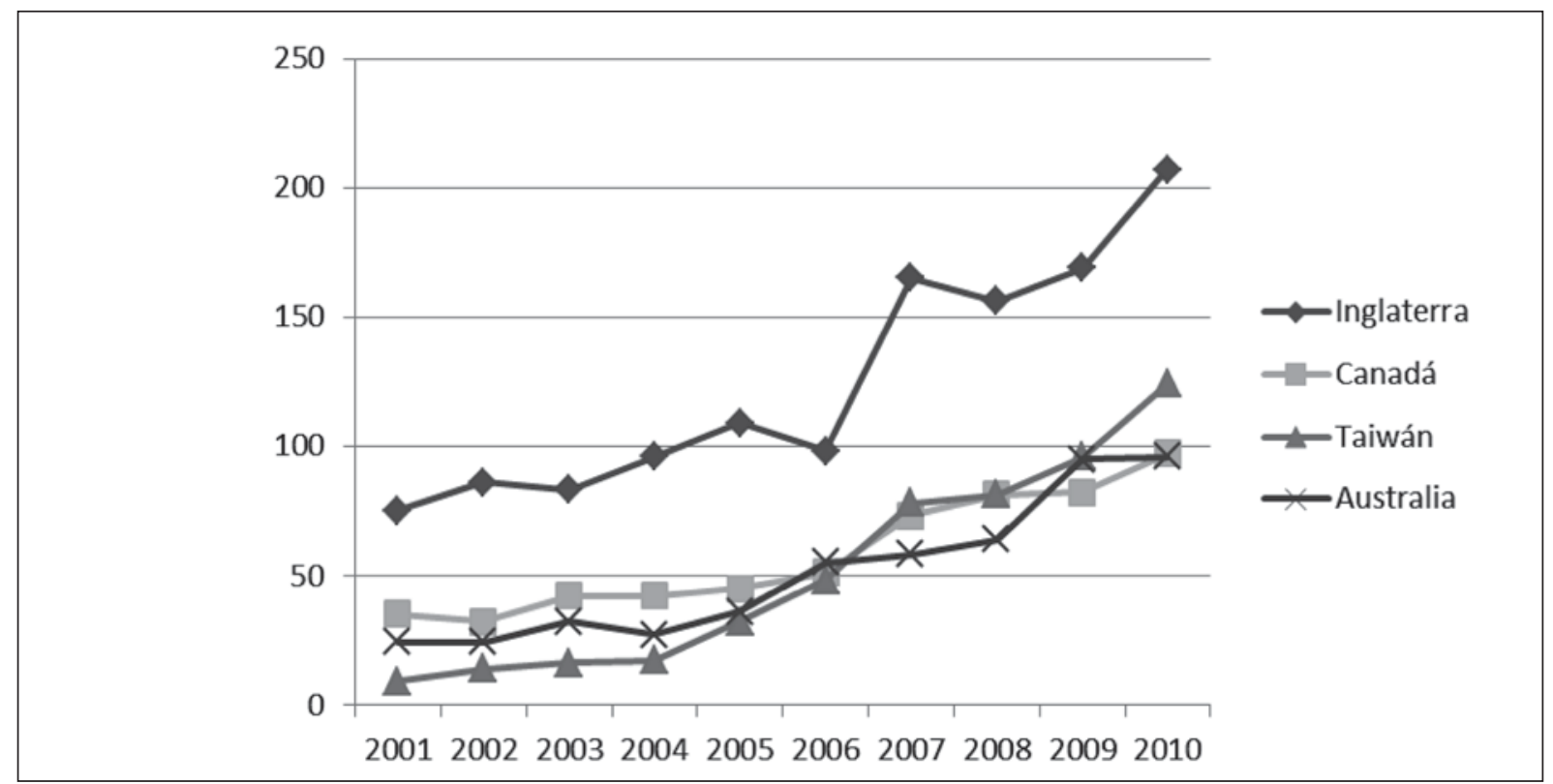

Figura 3. Evolución de la productividad científica de Inglaterra, Canadá, Taiwán y Australia, 2001-2010 Fuente: elaboración propia. 


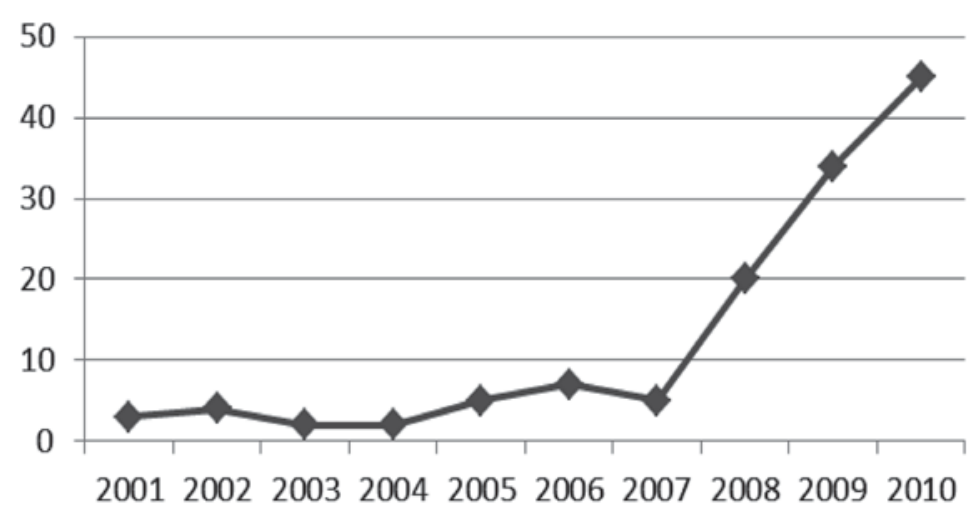

Figura 4. Evolución de la productividad científica de Brasil, 2001-2010

Fuente: elaboración propia.

al identificar que la brecha entre Estados Unidos y los otros países es extensa, se tomó la decisión de incluir la información en dos gráficos con el objetivo de proporcionar algunos patrones con mayor detalle.

En las figura 2 y 3 se muestran los países más productivos. Estos países marcan la pauta de la tendencia general en el número de publicaciones científicas, mostrada anteriormente en la figura 1. Como puede observarse en esta figura, Estados Unidos, que es el país con mayor número de publicaciones en el área y Australia, que es el quinto país más productivo, decrecieron en el número de publicaciones comprendidas en los años 2003 y 2004. Lo anterior, puede explicar el decrecimiento en la productividad global mostrada en la figura 1 . Sin embargo, otros países como Inglaterra, Canadá y Taiwán presentaron incrementos de productividad durante el 2003, que no tuvieron un efecto considerable contra la tendencia de Estados Unidos en este par de años.

Estados Unidos e Inglaterra tuvieron un decrecimiento entre los años 2007 y 2008, sin embargo, entre estos años el resto de países más productivos se mantuvieron constantes o incrementaron levemente su productividad, permitiendo que se presentara una tasa de crecimiento de publicación positiva del 6,8\%. Taiwán ha sido el país con mayor crecimiento en la productividad científica del área, posicionado como quinto país en el año 2001 y tercero en el año 2010 superando países como Canadá y Australia. Como puede comprobarse en las figuras 2 y 3 , la tendencia de productividad para los países mostrados es de incremento para los siguientes años.

Para el caso de Latinoamérica, la mayoría de países, exceptuando Brasil, aún no superan la barrera de seis artículos publicados por año. Su evolución muestra crecimientos y decrecimientos año tras año, aunque con una tendencia general de aumento. Por ejemplo, México presenta una tendencia de crecimiento notable desde el 2004 hasta el 2009, sin embargo, esta tendencia disminuye en el 2010. Este patrón de decrecimiento en el último año se aplica a Chile, Argentina, Venezuela y Colombia. Por otra parte, Brasil ha presentado una tasa de crecimiento elevada desde el 2007, donde se presenta un punto de inflexión con una tendencia creciente en la productividad científica (ver figura 4).

En la tabla 2 se muestran las 20 instituciones más productivas en el área de Gestión de la Tecnología. A pesar de que Estados Unidos es el país con mayor productividad científica en el tema, la institución más productiva se encuentra ubicada en Chi- 
Tabla 2. Lista de instituciones más productivas

\begin{tabular}{|c|l|c|l|}
\hline \# Registros & \multicolumn{1}{|c|}{ Instituciones } & \# Registros & Instituciones \\
\hline 51 & Hong Kong Polytech Univ, & $\mathbf{2 6}$ & Univ Warwick, \\
\hline 38 & Univ Melbourne, & $\mathbf{2 5}$ & Natl Univ Singapore, \\
\hline 33 & Univ Manchester, & $\mathbf{2 5}$ & Univ Washington, \\
\hline 30 & Natl Cheng Kung Univ, & $\mathbf{2 3}$ & Penn State Univ, \\
\hline 27 & Michigan State Univ, & $\mathbf{2 3}$ & Univ Calif Berkeley, \\
\hline 27 & MIT, & $\mathbf{2 3}$ & Univ Maryland, \\
\hline 27 & Monash Univ, & $\mathbf{2 3}$ & Univ Michigan, \\
\hline 27 & Nanyang Technol Univ, & $\mathbf{2 2}$ & Univ Leeds, \\
\hline 26 & Univ Cambridge, & $\mathbf{2 1}$ & City Univ Hong Kong, \\
\hline 26 & Univ Toronto, & $\mathbf{2 1}$ & Politecn Milan, \\
\hline
\end{tabular}

Fuente: elaboración propia.

na. Es pertinente destacar que las siguientes tres universidades más productivas están en Australia, Inglaterra y Taiwán respectivamente. La Universidad Politécnica de Hong Kong es la institución de educación superior que más ha contribuido en esta década de estudio con 51 publicaciones sobre la Gestión de la Tecnología. Las diferencias de productividad científica entre instituciones no se consideran tan notables como las encontradas entre países. Sin embargo, como era de esperarse, existe una relación entre la productividad a nivel institucional y la productividad en términos de países. Como puede observarse en la tabla 2, siete de las 20 instituciones son de Estados Unidos y cuatro pertenecen a Inglaterra. Por otra parte, es importante resaltar que, como puede observarse en la tabla 2 , no se encuentra ninguna institución latinoamericana o africana. Finalmente, es posible observar que, en países como Estados Unidos e Inglaterra, la productividad científica está más distribuida entre las instituciones en comparación a otros países donde se presenta centralización de la información en actores específicos (como en el caso de China).

La tabla 3 muestra los autores más productivos en el área. De los cinco más productivos; por ejemplo,
Lichtenthaler ha concentrado sus estudios principalmente en temas como la innovación abierta, transferencia tecnológica, licenciamiento y comercialización de tecnología. Ngai, por su parte, ha enfocado sus trabajos a revisiones de la literatura y al desarrollo de marcos de trabajo. Los es-

Tabla 3. Lista de autores más productivos en el área, 2001-2010

\begin{tabular}{|c|c|c|c|}
\hline 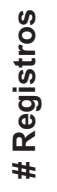 & $\begin{array}{l}\text { d } \\
\frac{0}{0} \\
\stackrel{0}{3}\end{array}$ & 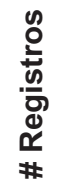 & $\begin{array}{l}\text { d } \\
\stackrel{0}{0} \\
\stackrel{ }{J}\end{array}$ \\
\hline 19 & Lichtenthaler, U & 14 & $\mathrm{Li}, \mathrm{Y}$ \\
\hline 18 & Ngai, E W T & 14 & McAdam, $\mathrm{R}$ \\
\hline 17 & Gottschalk, P & 14 & Rai, A \\
\hline 17 & Kauffman, R J & 14 & Sabherwal, R \\
\hline 17 & Keil, M & 14 & Tiwana, A \\
\hline 15 & Cheng, T C E & 13 & Lai, K H \\
\hline 15 & Lederer, A L & 13 & Lee, $\mathrm{H}$ \\
\hline 15 & Lin, C H & 13 & Lee, J \\
\hline 15 & Song, $\mathrm{M}$ & 12 & Irani, Z \\
\hline 14 & Gunasekaran, A & 12 & Pan, S L \\
\hline
\end{tabular}

Fuente: elaboración propia. 
tudios de Gottschalk, están asociados a la gestión del conocimiento. Kauffman, estudia dos grandes líneas: la teoría económica y análisis económicos; y una segunda línea de inversiones y servicios de las tecnologías de la información. Finalmente, Keil está centrado en la gestión de proyectos, riesgos de los proyectos y los procesos de tercerización. Las principales revistas donde diferentes autores publican sus investigaciones son: International Journal of Technology Management, Technovation, IEEE Transactions on Engineering Managament, Information Management y el International Journal of Operations \& Production Management.

\subsection{Análisis descriptivo y co-ocurrencia de palabras clave}

Las palabras clave utilizadas en las publicaciones ofrecen información sobre las tendencias de investigación desde el punto de vista de los autores [50]. Por ello, han sido utilizadas recientemente como unidad de análisis en diferentes estudios [51]. Al igual que el número de publicaciones, el número de palabras clave ha incrementado año tras año en la última década. La figura 5 muestra una comparación entre el número de artículos publicados por año y el número de palabras clave empleadas por los investigadores.

En la tabla 4 se observan los 20 temas por palabras clave más estudiados. En esta lista existen tres grandes grupos que marcan la pauta de la investigación en Gestión de la Tecnología, estos son: gestión de la innovación y del conocimiento; sistemas y tecnologías de la información y comunicación; y gestión de proyectos. Sin embargo, un tema recurrente en las diferentes investigaciones desarrolladas en el área es el estudio de casos, especialmente utilizado en temas emergentes.

La tendencia de los cinco temas más importantes durante los años 2001- 2010 puede ser observada en la figura 6. Aunque la gestión del conocimiento es el tópico mas estudiado, éste presentó un retroceso en el interés de los investigadores durante los años 2006 al 2008. También, puede ob-

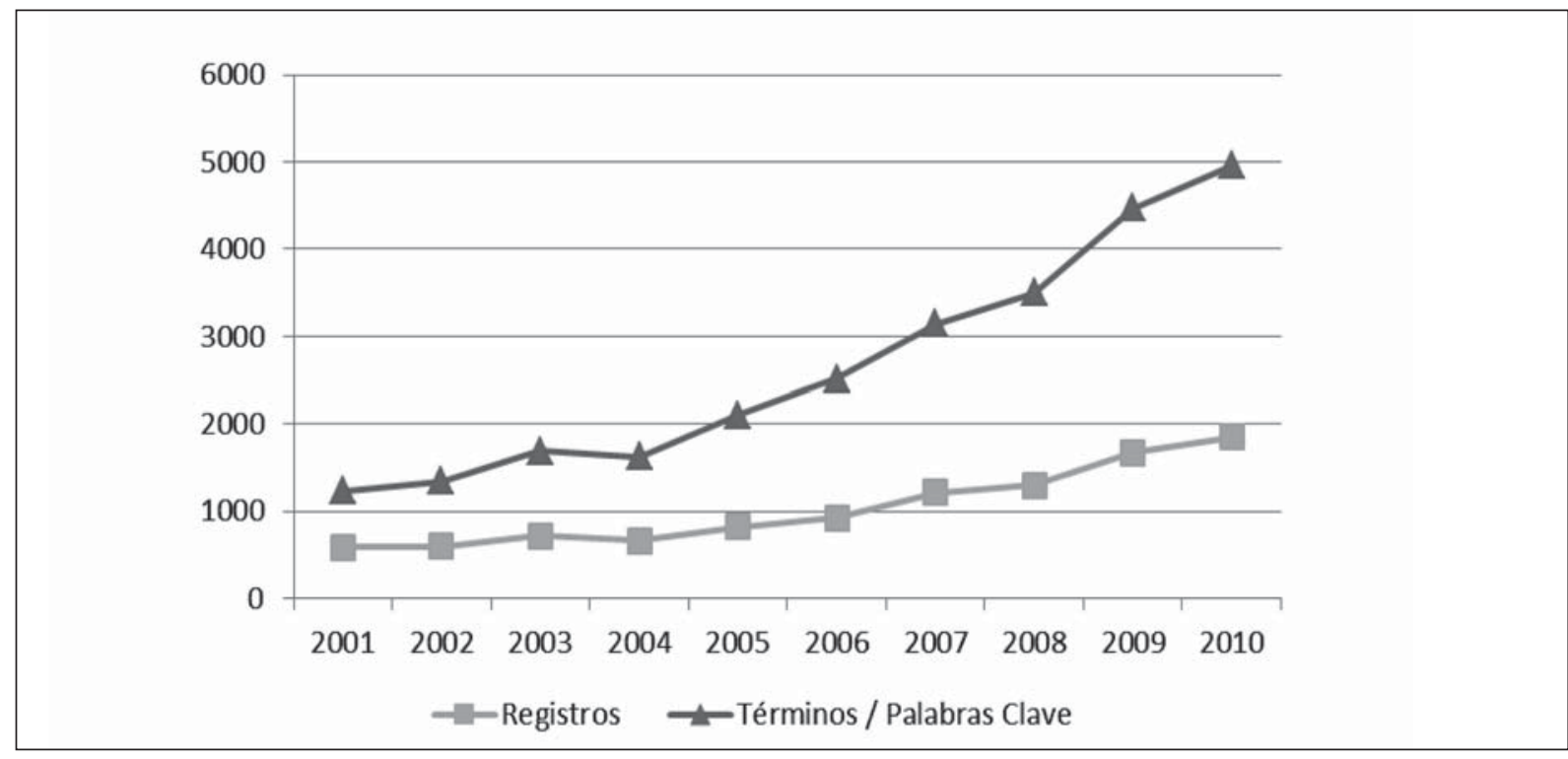

Figura 5. Comparación evolutiva entre el número de registros por año y el número de palabras clave empleadas por año

Fuente: elaboración propia. 
Tabla 4. Veinte temas por palabra clave más estudiados en las investigaciones de Gestión tecnológica

\begin{tabular}{|c|c|c|c|}
\hline \# Registros & Palabras Clave de Autor & \# Registros & Palabras Clave de Autor \\
\hline 457 & Gestión del Conocimiento & 113 & Tecnologías de Comunicación \\
\hline 355 & Innovación & 109 & China \\
\hline 311 & Cadena de Suministro & 108 & Director de proyectos \\
\hline 302 & Información tecnológica & 95 & Gestión informal \\
\hline 203 & Tecnología & 95 & Gestión del Riesgo \\
\hline 164 & Internet & 93 & Tercerización \\
\hline 150 & Sistema de Información & 91 & Desempeño \\
\hline 132 & Estudio de casos & 89 & Conocimiento compartido \\
\hline 123 & Directivo & $\mathbf{8 6}$ & Comunidad \\
\hline 114 & Directivos de tecnología & 74 & Transferencia de tecnología \\
\hline
\end{tabular}

Fuente: elaboración propia.

servarse que, en el último año analizado (2010); el tema ha decrecido considerablemente después de haber tenido un crecimiento en el periodo entre 2008 y 2009. Un dato importante a considerar, es el comportamiento del tema 'tecnologías de la información' que se ha mantenido en una tendencia creciente desde el 2005. Este comportamiento puede derivarse del auge de este tipo de tecnologías en la operacionalización de diferentes teorías de la ingeniería del conocimiento. Por tanto, se

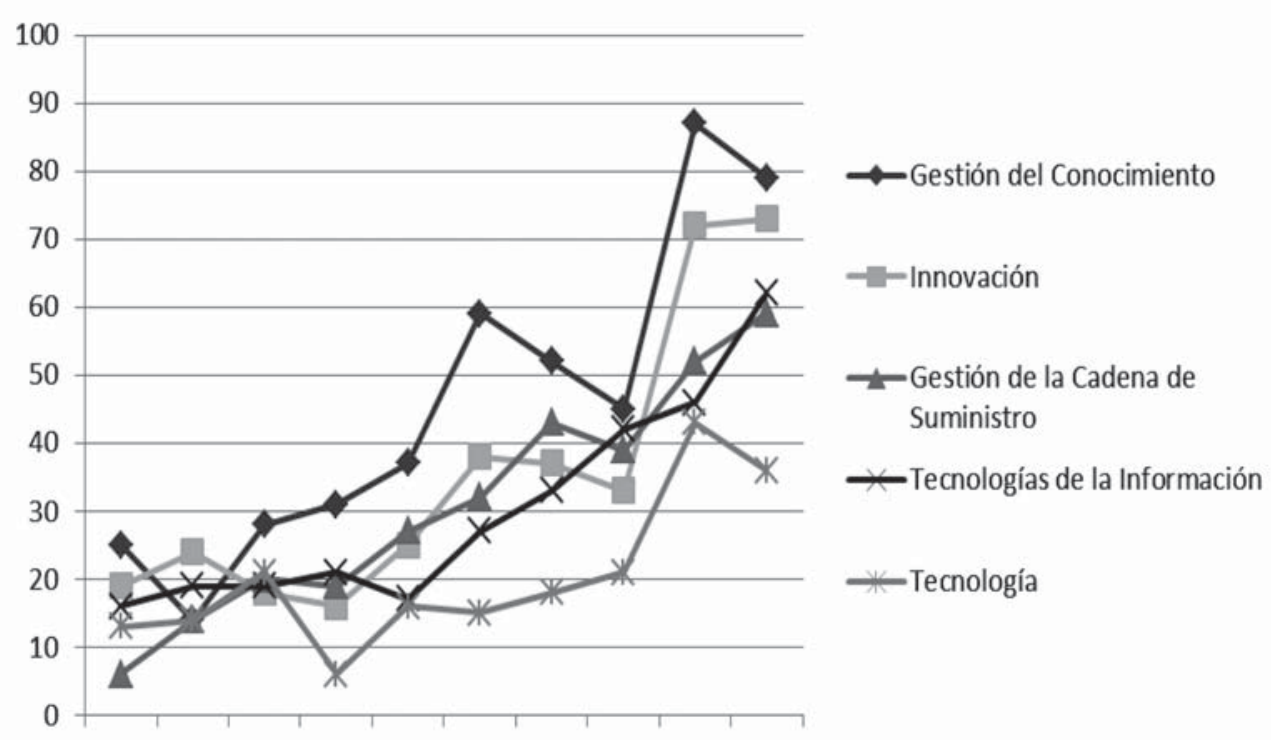

2001200220032004200520062007200820092010

Figura 6. Evolución de los cinco tópicos más estudiados en el área Fuente: elaboración propia. 
observa una orientación de los investigadores a la aplicación de las diferentes teorías, especialmente las relacionadas con la gestión del conocimiento y la innovación, que han sido de especial interés en los últimos años.

Con el propósito de obtener la estructura de conocimiento del área Gestión de la Tecnología, se utilizaron las 100 palabras clave más utilizadas por los autores para realizar el análisis de co-ocurrencia. Esta investigación acepta las premisas de Bhattacharya y otros, (2003) quienes argumentan que, dada la naturaleza de las palabras clave, estas son un elemento fundamental del conocimiento en un área de investigación específica. La figura 7 muestra la estructura intelectual de los conceptos principales investigados en la gestión de la tecno- logía basado en las palabras clave que los autores asignaron a sus investigaciones.

El mapa fue realizado aplicando la técnica de Visualización de Similaridades-VOS. Esta técnica permite dibujar una gráfica bidimensional de la proximidad entre palabras en un espacio conceptual de un área específica. En el mapa, entre más grande es el número de ítems o términos en el vecindario de algún punto especifico, más grande es el tamaño de las palabras y el punto es resaltado en color negro. La localización de las palabras clave en el espacio de conocimiento es una representación de similaridad (número de veces que un par de palabras han sido utilizadas simultáneamente en los artículos); el tamaño de la palabra clave es un indicador de su frecuen-

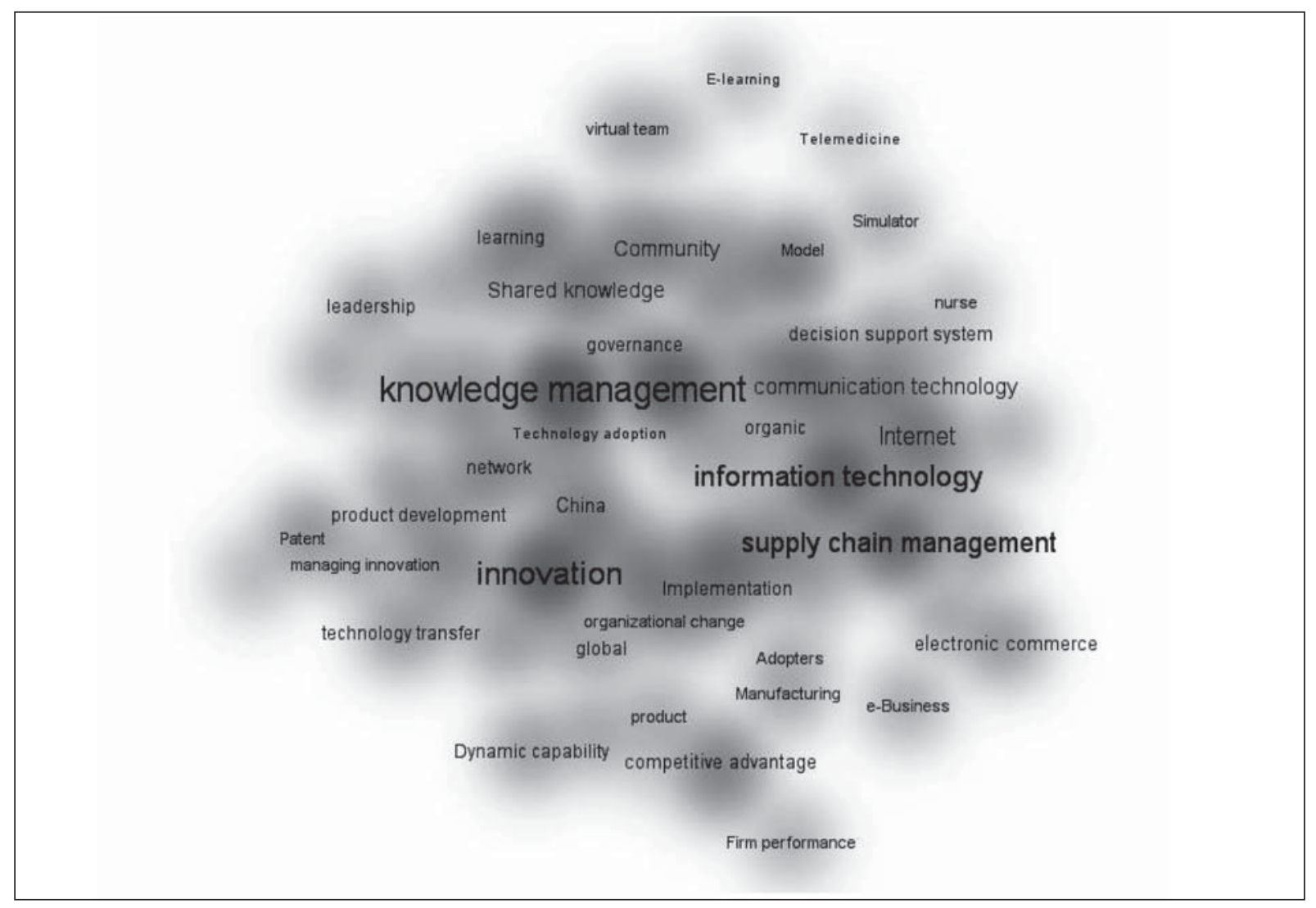

Figura 7. Mapa de la estructura intelectual de la investigación en Gestión de la Tecnología Fuente: elaboración propia. 
cia en la literatura científica estudiada. Por tanto, el mapa ofrece información relacionada a temas centrales como: gestión del conocimiento, innovación y tecnologías de la información; además, muestra las relaciones entre tópicos de investigación estudiados. Así, en la figura se observa que la gestión de conocimiento ha sido estudiada junto a tópicos como gobierno, adopción tecnológica, conocimiento compartido y liderazgo. Entre los temas más estudiados es importante resaltar la corta distancia en el espacio de conocimiento entre los tópicos tecnologías de la información y gestión de la cadena de suministro. Lo anterior, indica que varias de las investigaciones en Gestión de la Tecnología han estudiado conjuntamente estos dos tópicos al igual que otros conceptos con menor intensidad en el mapa como internet, implementación y comercio electrónico. Por otra parte, el tema de innovación ha sido investigado conjuntamente con temas como: desarrollo de productos, transferencia tecnológica, cambio organizativo y patentes.

Finalmente, las palabras clave fueron clasificadas para obtener información acerca del año en que ellas aparecieron en las diferentes publicaciones durante los años comprendidos en el periodo de los años 2001-2010. Esta información revela temas que están emergiendo en la investigación y que pueden servir como tema de interés de nuevas investigaciones y nuevas perspectivas de investigación en el área. Entre estos temas se encuentran: e-learning, capacidad de absorción de conocimiento (aunque esta capacidad es un constructo que se originó en 1990), librerías digitales, desempeño de negocios, cambio climático, sistemas electrónicos RFID, capital humano, creación e conocimiento, servicios web, entre otros. Otros tópicos emergentes más recientes son: auto-eficacia, gestión de procesos del negocio (BPM), intercambio electrónico de datos, éxito en los sistemas de información, practicas, mercado B2B y orientación al cliente.

\section{CONCLUSIONES}

Este artículo ha realizado un estudio bibliométrico de la disciplina Gestión de la Tecnología, aplicando herramientas descriptivas y análisis de co-ocurrencia de palabras clave en los artículos publicados entre los años 2001-2010. El análisis de la publicación de artículos sugiere un crecimiento en la tendencia de productividad científica en los años futuros. La mayoría de las investigaciones desarrolladas sobre la gestión de la tecnología son de carácter multidisciplinar por la diversidad de áreas estudiadas en la última década. Las áreas con mayor dominio en la disciplina son: economía y negocios, ciencias de la computación, ingeniería, ciencias de la información y ciencias de la gestión.

Al igual que en otras disciplinas, la actividad investigativa se ha concentrado en países norteamericanos y europeos. Existiendo una reducida participación de países latinoamericanos y africanos. También es importante destacar el incremento en la productividad científica de Brasil desde el año 2007 y de Taiwán, especialmente en la última década. Los otros países deberían generar entornos más proactivos y fomentar la creación de estrategias para el incremento y fortalecimiento de investigaciones en esta disciplina.

La Gestión de la Tecnología ha empezado a crear su propia literatura, lo cual le ha permitido generar una reputación en la comunidad académica. Lo anterior, se ve reflejado en el número de publicaciones incluidas en revistas creadas como estrategia de difusión de las investigaciones en la disciplina: International Journal of Technology Management, Technovation, y IEEE Transactions on Engineering Managament. La investigación se ha enfocado principalmente en las siguientes líneas de investigación: gestión del conocimiento y la innovación, tecnologías de la información y la comunicación. Lo anterior, hace latente la transición de las diferentes teorías a prácticas específicas que puedan ser utilizadas por diferentes actores. 


\section{AGRADECIMIENTOS}

Los aportes de esta investigación fueron consolidados por los resultados de la investigación derivada del proyecto títulado: Una Estructura
Multidimensional de la Capacidad de Innovación financiado por la Vicerrectoria de Investigación y Extensión de la Universidad Industrial de Santander.

\section{Referencias}

[1] A. Pilkington and T. Teichert, "Management of technology: themes, concepts and relationships", Technovation, Vol. 26, No. 3, pp. 288-299, 2006.

[2] W. B. Zhener II, "The Management of Technology (MOT) Degree: A Bridge between Technology and Strategic Management", Technology Analysis and Strategic Management, Vol. 12, No. 2, pp. 283-291, 2000.

[3] J. D. Linton, "Perspective: Ranking Business School on the Management of Technology", Journal of Product Innovation Management, Vol. 21, No. 6, pp. 416-430, 2004.

[4] A. Pritchard, "Statistical bibliography or bibliometrics", Journal of Documentation, Vol. 25, No. 4, pp. 348-349. 1969.

[5] I. K. Ravichandra, Quantitative Methods for Library and Information Science, New York: Wiley, 1983.

[6] A. Rip and J. P. Courtial, "Co-Word Maps of Biotechnology: An Example of Cognitive Scientometrics", Scientometrics, Vol. 6, No. 6, pp. 381-400, 1984.

[7] T. U. Daim, G. Rueda, H. Martin and P. Gerdsri, "Forecasting emerging technologies: Use of bibliometrics and patent analysis", Technological Forecasting and
Social Change, Vol. 73, No. 8, pp. 9811012, 2006.

[8] B. Durisin, G. Calabretta and V. Parmeggiani, "The Intellectual Structure of Product Innovation Research: A Bibliometric Study of the Journal of Product Innovation Management 1984-2004", Journal of Product Innovation Management, Vol. 27, No. 3, pp. 437-451, 2010.

[9] K. Artto, M. Martinsuo, H. G. Gemunden and J. Murtoaro, "Foundations of program management: A bibliometric view", International Journal of Project Management, Vol. 27, No.1, pp. 1-18, 2009.

[10] W. Raghupathi and S. Nerur, "Research Themes and Trends in Health Information Systems", Methods of information in medicine, Vol. 47, No. 5, pp. 435-442, 2008.

[11] M. Tsay, "A bibliometric analysis of hydrogen energy literature, 1965-2005", Scientometrics, Vol. 75, No. 3, pp. 421-438, 2008 .

[12] F. Lufrano and P. Staiti, "A Bibliometric Analysis of the International Literature in Super capacitors", International Journal of Electrochemical Science, Vol. 4, No. 5, pp. 173-186, 2009.

[13] M. E. Falagas, A. S. Michalopoulos, I. A. Bliziotis and E. S. Soteriades, "A bibliome- 
tric analysis by geographic area of published research in several biomedical fields. 1995-2003", Canadian Medical Association Journal, Vol. 175, No. 11, p.1389, 2006.

[14] A. F. J. Van Raan, "Advanced bibliometric methods as quantitative core of peer review based evaluation and foresight exercises", Scientometrics, Vol. 36, No. 3, pp. 397-420, 1996.

[15] E. Garfield, "Citation analysis as a tool in journal evaluation", Science, Vol. 178, No. 4060, pp. 471-479, 1972.

[16] D. De Solla Price, Little science, big science, New York: Columbia University Press, 1963.

[17] P. Palvia, D. Leary, E. Mao, V. Midha, P. Pinjani and A. F. Salam, "Research methodologies in MIS: An update", Communications of the Association for Information Systems, Vol. 14, No. 24, pp. 526542, 2004.

[18] P. Palvia, E. Mao, A. F. Salam and K. S. Soliman, "Management information systems research: What's there in a methodology?", Communications of the Association for Information Systems, Vol. 11, No. 16, pp. 289-309, 2003.

[19] D. T. Hawkins, "Unconventional uses of on-line information retrieval systems: Online bibliometric studies", Journal of the American Society for Information Science, Vol. 28, No. 1, pp. 13-18, 1977.

[20] A. Pritchard, "Statistical bibliography or bibliometrics", Journal of Documentation, Vol. 25, No. 4, pp. 348-349, 1969.

[21] D. W. Aksnes and R. E. Taxt, "Peers reviews and bibliometric indicators: A com- parative study at Norwegian University", Research Evaluation, Vol. 13, No. 1, pp. 33-41, 2004.

[22] C. A Estabrooks, C. Winther and L. Derksen, "Mapping the field: a bibliometric analysis of the research utilization literature in nursing", Nursing Research, Vol. 53, No. 5, pp. 293-303, 2004.

[23] J. Keiser and J. Utzinger, "Trends in the core literature on tropical medicine: A bibliometric analysis from 1952-2002" , Scientometrics, Vol. 62, No. 3, pp. 351365, 2005.

[24] R. Tang and M. Thelwall, "US academic departmental Web-site interlinking in the United States disciplinary differences", Library \& Information Science Research, Vol. 25, No. 4, pp. 437-458, 2003.

[25] F. Murray, "Innovation as co-evolution of scientific and technological networks: Exploring tissue engineering", Research Policy, Vol. 31, pp. 1389-1403, 2002.

[26] . Cahlik, "Search for fundamental articles in economics", Scientometrics, Vol. 49, No. 3, pp. 389-402, 2000.

[27] A. Cambrosio, C. Limoges, J. P. Courtial and F. Laville, "Historical scientometrics? Mapping over 70 years of biological safety research with coword analysis", Scientometrics, Vol. 27, No. 2, pp. 119-143, 1993.

[28] Y. Ding, G. G. Chowdhury, and S. Foo, "Bibliometric cartography of information retrieval research by using co-word analysis", Information Processing \& Management, Vol. 37, No. 6, pp. 817-842, 2001.

[29] B. Lee and Y. Jeong, "Mapping Korea's national $R \& D$ domain of robot technology 


\section{revisión}

by using the co-word analysis", Scientometrics, Vol. 77, No. 1, pp. 3-19, 2008.

[30] M. W. Neff and E. A. Corley, "35 years and 160,000 articles: A bibliometric exploration of the evolution of ecology", Scientometrics, Vol. 80, No. 3, pp. 657-682, 2009.

[31] M. I. Viedma-Del-Jesus, P. Perakakis, M. A. Muñoz, A. G. López-Herrera, and J. Vila, "Sketching the first 45 years of the journal Psychophysiology (1964-2008): A co-word-based analysis", Psychophysiology, Vol. 48, No. 8, pp.1029-1036, 2011.

[32] J. Stegmann and G. Grohmann, "Hypothesis generation guided by co-word clustering", Scientometrics, Vol. 56, No. 1, pp. 111-135, 2003.

[33] Q. He, "Knowledge discovery through coword analysis", Library Trends, Vol. 48, No. 1, pp.133-159, 1999.

[34] J. P. Courtial, M. Callon and M. Sigogneau, "Is indexing trustworthy? Classification of articles through co-word analysis", Journal of Information Science, Vol. 9, No. 2, pp. 47-56, 1984.

[35] R. R. Braam, H. F. Moed and F. J. van Raan, "Mapping of science by combined cocitation and word analysis II. Dynamical aspects", Journal American Society Information Science, Vol. 42, No. 4, pp. 252266, 1991.

[36] L. Vaughan and J. You, "Word co-occurrences on Webpages as a measure of the relatedness of organizations: A new Webometrics concept", Journal of Informetrics, Vol. 4, No. 4, pp. 483-491, 2010.

[37] Y. Tseng, C. Lin and Y. Lin, "Text mining techniques for patent analysis", Informa- tion Processing \& Management, Vol. 43, No. 5, pp. 1216-1247, 2007.

[38] N. J. Eck and L. Waltman, "VOS: A New Method for Visualizing Similarities Between Objects", Advances in Data Analysis, pp. 299-306, Springer Berlin Heidelberg, 2007

[39] A. J. Izenman, "Multidimensional Scaling and Distance Geometry", Modern Multivariate Statistical Techniques, pp. 463-504, Springer New York, 2008.

[40] W. Chiu and Y. Ho, "Bibliometric analysis of homeopathy research during the period of 1991 to 2003", Scientometrics, Vol. 63, No. 1, pp. 3-23, 2005.

[41] M. Rahman, T. L. Haque and T. Fukui, "Research articles published in clinical radiology journals: Trend of contribution from different countries", Academic Radiology, Vol. 12, No. 7, pp. 825-829, 2005.

[42] A. L. Dannenberg, "Use of epidemiology in medical specialties: An examination by citation analysis", American Journal of Epidemiology, Vol. 121, No. 1, pp. 140$151,1985$.

[43] R. Rajendram, G. Lewison and V. R. Preedy, "Worldwide alcohol-related research and the disease burden", Alcohol and Alcoholism, Vol. 41, No. 1, pp. 99-106, 2006.

[44] J. C. Davis and J. G. Gonzalez, "Scholarly journal articles about the Asian Tiger Economies: Authors, journals and research fields, 1986-2001", Asian-Pacific Economic Literature, Vol. 17, No. 2, pp. 51-66, 2003.

[45] S. Bhattacharya, H. Kretschmer, and M. Meyer, "Characterizing intellectual spaces 
between science and technology", Scientometrics, Vol. 58, No. 2, pp. 369-390, 2003.

[46] H. Schildt, "SITKIS: Software for Bibliometric Data Management and Analysis", Helsinki: Institute of Strategy and International Business, 2002.

[47] N. J. van Eck, L. Waltman, R. Dekker and J. van der Berg, "A comparison of two techniques for bibliometric mapping: Multidimensional scaling and VOS", Journal of the American Society for Information Science and Technology, Vol. 61, No. 12, pp. 2405-2416, 2010.

[48] R. J. W. Tijssen, "Discarding the "basic science/applied science" dichotomy: A knowledge utilization triangle classification system of research journals", Journal of the American Society for Information Science and Technology, Vol. 61, No. 9, pp. 1842-1852, 2010.

[49] S. Arunachalam and M. Jinandra, "Mapping international collaboration in science in Asia through coauthorship analysis", Current Science, Vol. 79, No. 5, pp. 621$628,2000$.

[50] E. Garfield, "Keywords plus-ISIS breakthrough retrieval method. 1. Expanding your searching power on current-contents on diskette", Current Contents, Vol. 32, pp. 5-9, 1990.

[51] W. Chiu and Y. Ho, "Bibliometric analysis of tsunami research", Scientometrics, Vol. 73, No. 1, pp. 3-17, 2007. 